\title{
CMOS sensors with high resistivity epitaxial layer
}

\author{
A. Besson ${ }^{a}$, M. Winter ${ }^{a}$, J. Baudot ${ }^{a}$, G. Bertolone ${ }^{a}$, N. Chon-Sen ${ }^{a}$, G. Claus $^{a}$, \\ C. Colledani ${ }^{a}$, Y. Degerli ${ }^{b}$, A. Dorokhov ${ }^{a}$, G .Dozière ${ }^{a}$, W. Dulinski ${ }^{a}$, M. Gelin ${ }^{a}$, \\ A. Geromitsos ${ }^{a}$, M. Goffe ${ }^{a}$, A. Himmi ${ }^{a}$, C. Hu-Guo ${ }^{a}$, K. Jaaskelainen ${ }^{a}$, F. Morel ${ }^{a}$,

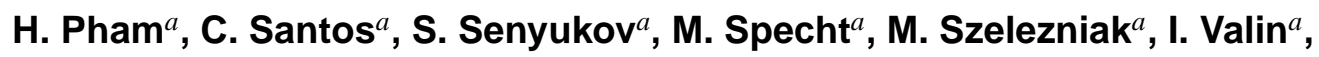 \\ G. Voutsinas ${ }^{a}$. \\ ${ }^{a}$ IPHC/IN2P3/CNRS - Université de Strasbourg \\ Strasbourg, France \\ ${ }^{b}$ IRFU/SEDI - CEA \\ Saclay, France
}

E-mail: abesson@in2p3.fr, marc.wintereiphc.cnrs.fr

CMOS Pixel Sensors (CPS) are foreseen to equip vertex detectors where priority is given to granularity, material budget and power consumption, potentially at the expense of read-out speed and radiation tolerance. Being initially developed for an experiment at the ILC, the sensors came out to be well suited to Heavy Ion Collision experiments (STAR at RHIC, CBM at FAIR, ...), and their intrinsic potential offers attractive perspectives for the vertex detector to be operated at the SuperB factory. Another trend motivating their continuous development concerns trackers, where granularity is less an issue but material budget, power consumption and fabrication costs may be significantly reduced when using CMOS pixel sensors instead of usual semi-conducting devices. For many years, CPS were manufactured with commercial wafers featuring exclusively low resistivity (i.e. typically $10 \Omega \cdot \mathrm{cm}$ ) epitaxial layers. The interest of industry for high resistivity epitaxial layers is a rather recent event, with a considerable impact on the potential of the CPS (e.g. a typical signal-to-noise ratio of about 35-40). Several sensors were fabricated since early 2010 with a $>400 \Omega \cdot \mathrm{cm}$ resistivity epitaxial layer, available in a $0.35 \mu \mathrm{m}$ process, and tested on particle beams.

This article illustrates the maturity and the potential of the technology, first by presenting the first vertex detector equipped with CPS (STAR-HFT upgrade), then by providing insight of the next steps of the R\&D, which are based on an emerging CMOS technology using a $0.18 \mu \mathrm{m}$ feature size, and aim at a large area beam telescope for the EU-FP7 project AIDA. Moreover, the development of a very light ladder, equipped on both faces with $50 \mu \mathrm{m}$ thin sensors, will be overviewed.

XXIst International Europhysics Conference on High Energy Physics

21-27 July 2011

Grenoble, Rhône-Alpes France

\footnotetext{
* Speaker.
} 


\section{Introduction}

CMOS Pixel Sensors (CPS)[1], have been developed during the last twelve years for the advent of a new generation of vertex detectors, where priority is given to granularity, material budget and power consumption. A particularly attractive aspect of CPS is their thin sensitive volume (typically 10-20 $\mu \mathrm{m}$ ). This feature, combined with the possibility to operate the sensors at room temperature with moderate cooling, allows for very low material budget devices. Moreover, their high granularity resulting into excellent, micron level, spatial resolution, the devices provide very accurate tracking performances.

Analog processing of the signal (preamplification, pedestal subtraction) takes place in the pixel itself. For the sake of granularity and power consumption, the mixed and digital signal processing micro-circuits complementing the signal processing (charge encoding, sparsification, etc.) are integrated on the sensor substrate at the chip periphery. The chip read-out is then based on a rolling shutter architecture where the pixels are organised in columns read out in parallel. This share of functionnalities is a basic feature of the MIMOSA sensors developed by the IPHC-IRFU collaboration [2].

Despite the sequential, row by row, read-out of the rolling shutter approach, the $\lesssim 200 \mathrm{~ns}$ row read-out time allows for full scale sensor read-out times in a regime (in the ordre of 10-100 $\mu s$ ) suited to a wide range of applications where the low power consumption $(\lesssim 1 \mu \mathrm{W} / \mathrm{pixel})$ is a major issue.

This article starts by exposing the production of the CPS, called ULTIMATE, for the STARHFT at RHIC [3], which will be the first vertex detector equipped with this type of sensors. It next summarises the test results of the ULTIMATE sensor prototype. The second part of the paper provides an insight of the next steps of the R\&D, which are based on an emerging CMOS technology using a $0.18 \mu \mathrm{m}$ feature size, and should allow realising a large area beam telescope for the EU-FP7 project AIDA [4] using stitching. The last part presents the state-of-the-art of CPS based pixellated systems, developed within the PLUME project [5], where a very light ladder is being worked out, equipped on both faces with $50 \mu \mathrm{m}$ thin sensors.

\section{STAR-HFT Vertex Detector Upgrade}

The first vertex detector equipped with CPS is currently being built for the STAR experiment at RHIC. Named STAR-PXL, it is composed of two cylindrical layers, with radii of 2.5 and $8 \mathrm{~cm}$ (see figure 1). It is built from 40 ladders, each made of 10 sensors and featuring a total material budget of $0.37 \% X_{0}$ per layer. The 400 sensors equipping the detector correspond to $\simeq 370$ Mpixels.

The Ultimate sensor, designed in the AMS-0.35 $\mu \mathrm{m}$-OPTO technology, comprises $928 \times$ 960 pixels with $20.7 \mu \mathrm{m}$ pitch and a $15 \mu \mathrm{m}$ thick, high resistivity $(>400 \Omega \cdot \mathrm{cm})$ epitaxial layer, and includes radiation tolerant structures. Its power consumption amounts to $\lesssim 135 \mathrm{~mW} / \mathrm{cm}^{2}$, corresponding to $\sim 0.8 \mu \mathrm{W}$ per pixel. The read-out time following from the rolling shutter architecture is $<200 \mu \mathrm{s}$. The sensor delivers a binary signal based on discriminators ending each of the 928 columns followed by a zero suppression micro-circuitry. It is suited to a flux of $10^{6}$ particles $/ \mathrm{cm}^{2} / \mathrm{s}$.

The sensor has been tested with a $120 \mathrm{GeV} / \mathrm{c}$ pion beam at the CERN-SPS in July 2011, in running conditions approaching those of the STAR-PXL (e.g. T $=30^{\circ} \mathrm{C}, 150 \mathrm{kRad}$ ionising dose). 

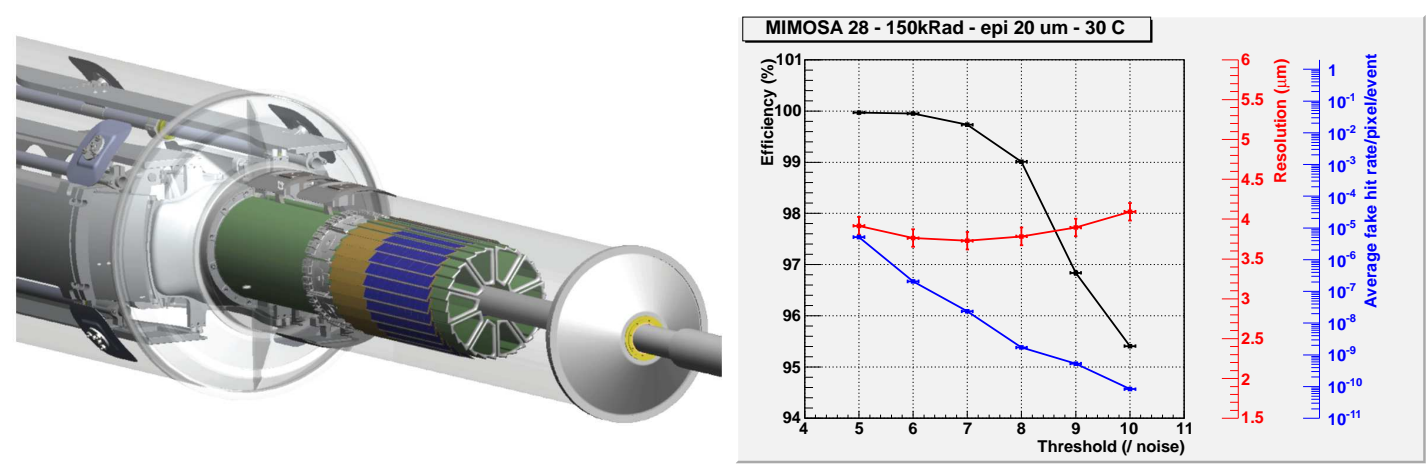

Figure 1: Left: STAR-PXL 3D view (ladders appear in blue). Right: Ultimate Sensor performances. The efficiency (black), resolution (red) and fake rate (blue) are shown versus the discriminator threshold.

Figure 1 summarises the performances observed. The chip reaches a detection efficiency $\gtrsim 99.9 \%$ even for high discriminator threshold values, where the fake hit rate (due to pixel noise fluctuations) is below $10^{-6}$. Providing moreover a spatial resolution of $\simeq 3.7 \mu \mathrm{m}$, the sensor is proven to meet the most important STAR-PXL requirements.

Several extensions of UltimATE for other experiments are planned. Their requirements are however difficult to satisfy with the CMOS $0.35 \mu \mathrm{m}$ process because of its intrinsic limitations. The modest number of metallisation layers and the feature size itself, which constrains the microcircuits density and is suspected to weaken the ionising radiation tolerance, are among the most limiting factors, and do not allow to fully exploit the potential of the CPS technology. Furthermore, the process only allows using NMOS transistors inside the pixels, unless parasitic charge collection occurs. To adapt the sensors to requirements of upcoming applications, a more advanced manufacturing technology had to be considered, based on a $0.18 \mu \mathrm{m}$ feature size.

\section{Toward an extension of application domains}

It is only since the last few years that semi-conductor industry offers a $0.18 \mu \mathrm{m}$ (imaging) process featuring manufacturing parametres expected to be well suited to subatomic physics experiments. Besides the benefits coming from the feature size, the technology offers 6 to 7 metallisation layers and a deep P-well option allowing for PMOS transistors inside the in-pixel micro-circuitry. Moreover, the epitaxial layer resistivity may amount to several $\mathrm{k} \Omega \cdot \mathrm{cm}$. All these new features are expected to improve significantly the global performances of CPS and to make them reach the signal processing capability and radiation hardness required by several projects motivating their R\&D (CBM at FAIR, ALICE-ITS upgrade, SuperB, ILD [2] for ILC, etc.).

Another interesting feature offered by this technology is stitching, which allows for sensors with multi-reticule dimensions. The AIDA (EU-FP7 WP 9.3) project [4], which is supposed to provide a large area beam telescope coupled to a target and a vertex detector sector, will fully benefit from this progress. It will also allow testing the double-sided ladders described in the next section. 


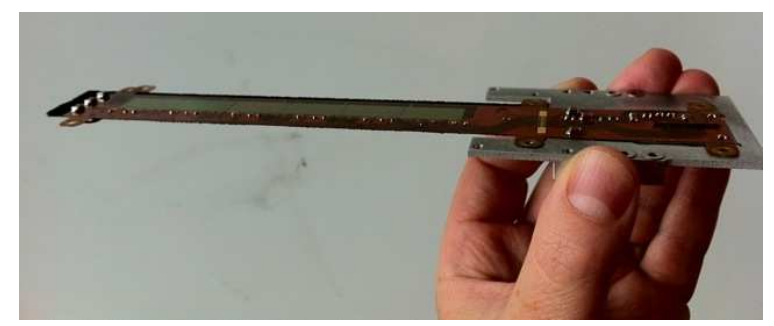

Figure 2: Picture of the PLUME prototype

\section{Mechanical integration and material budget}

In ordre tu fully exploit the advantages of CPS, great care is to be given to their system integration. The PLUME (Pixelated Ladder with Ultra low Material Embedding) project [5] has been initiated in order to establish a proof of principle of a CPS based double-sided ladder for the ILD vertex detector [2]. It consists in realising a ladder populated with 6, $50 \mu \mathrm{m}$ thin, CPS on each side, connected to a low mass flex cable and supported by a silicon carbide foam (see figure 2). The full ladder features 8.4 Million pixels read out in $\sim 100 \mu \mathrm{s}$, resulting into $\simeq 80 \mathrm{Mbits} / \mathrm{s}$. The material budget reached by this first prototype is $\simeq 0.6 \% X_{0}$ on the active area. The ladder will be tested at the CERN-SPS with minimum ionising particles in November 2011. A second generation prototype, nearly twice thinner, is being designed, using a narrower flex and a lower mass support. It will feed the AIDA project component studying the added value of ultra-light double-sided ladders.

\section{Summary}

After 12 years of $R \& D$, the CPS are reaching the phase of their first mature stage, where the specifications of devices requiring highly granular and thin, low power, sensors (vertex detectors, telescopes, etc.) can be equipped. For the mid-term, a new R\&D programme has been launched, aiming for significant performance improvements using a $0.18 \mu \mathrm{m}$ CMOS imaging process, in ordre to comply with more demanding applications (e.g. ALICE and CBM vertex detectors). This $\mathrm{R} \& \mathrm{D}$ is accompanied by the development of ultra-light ladders allowing to fully exploit the advantages of the CPS.

\section{References}

[1] R. Turchetta et al., A Monolithic Active Pixel Sensor for Charged Particle Tracking and Imaging Using Standard VLSI CMOS Technology, Nucl.Instr.Meth. in Phys. Res. A458 (2001) 677-689.

[2] G. Voutsinas et al., Studies for a $10 \mu \mathrm{s}$, thin, high resolution CMOS pixel sensor for future vertex detectors, Nucl. Phys. B - Proc. Suppl. 215 (2011) 48-50, and references therein.

[3] M. Szelezniak et al., MAPS Based Vertex Detector At STAR, PoS VERTEX2010 (2010) 043.

[4] AIDA project, http://aida.web.cern.ch/aida/index.html.

[5] A. Nomerotski et al., PLUME collaboration: Ultra-light ladders for linear collider vertex detector, Nucl.Instr.Meth. A650 (2011) 208-212. see also http://www.iphc.cnrs.fr/PLUME.html. 